Cahiers de recherches médiévales

Journal of medieval studies

$8 \mid 2001$

La protection spirituelle au Moyen Âge

\title{
La Trinité à l'heure de la mort
}

Sur les motifs trinitaires en contexte funéraire à la fin du Moyen Âge (m. XIV ${ }^{\mathrm{e}}$-déb. $\mathrm{XVI}^{\mathrm{e}}$ siècle)

\section{François Bœspflug}

\section{CpenEdition}

Journals

Édition électronique

URL : https://journals.openedition.org/crm/389

DOI : $10.4000 / \mathrm{crm} .389$

ISSN : $1955-2424$

Éditeur

Honoré Champion

Édition imprimée

Date de publication : 15 janvier 2001

Pagination : 87-106-xi

ISSN : 1272-9752

\section{Référence électronique}

François Bœspflug, "La Trinité à l'heure de la mort 》, Cahiers de recherches médiévales [En ligne], 8 |

2001, mis en ligne le 13 mars 2008, consulté le 15 décembre 2022. URL : http://

journals.openedition.org/crm/389; DOI : https://doi.org/10.4000/crm.389

Ce document a été généré automatiquement le 15 décembre 2022.

Tous droits réservés 


\section{La Trinité à l'heure de la mort}

Sur les motifs trinitaires en contexte funéraire à la fin du Moyen Âge (m. XIV ${ }^{\mathrm{e}}$-déb. XVI ${ }^{\mathrm{e}}$ siècle)

\section{François Bœspflug}

1 L'obsession de la mort, à la fin du Moyen Âge, fut assez forte pour toucher toutes les couches de la société et s'exprimer dans tous les domaines de la culture : à preuve, dans le domaine de la liturgie, le développement de l'office des morts, dont témoignent les livres d'heures ${ }^{1}$, et la multiplication, à partir de la seconde moitié du XIV siècle notamment, des messes pour les défunts ${ }^{2}$; et, dans le domaine de l'art, entre autres manifestations, l'irruption du macabre ${ }^{3}$ et la montée en puissance du pathétique, aussi bien dans l'art funéraire que dans les manuscrits à peintures. Certaines miniatures condensent cette évolution, telle la miniature poignante illustrant l'office des morts dans les Grandes Heures de Rohan, avec Dieu le Père se penchant du ciel vers la dépouille d'un mort gisant à terre, ou cette autre miniature, de même structure que la précédente, montrant Dieu le Père se penchant vers le corps du Christ descendu de Croix et posé à même le sol, avec saint Jean tournant la tête comme pour adresser des reproches à Dieu, tout en retenant la Vierge ${ }^{4}$.

Pour combattre le pénible sentiment d'insécurité créé par la pensée de la mort et du jugement, diverses conduites ont été adoptées, parmi lesquelles vient en bonne place la commande d'images anticipant la comparution devant le Juge suprême et visant à donner à celle-ci, d'une manière ou d'une autre, et autant que faire se pouvait, une issue heureuse, vécue comme rassurante. Or, la figure du Juge, sur certaines de ces images, va se complexifier jusqu'à faire apparaître la Trinité elle-même. Dans le cadre de mes recherches sur la Trinité dans l'art médiéval d'Occident, je relève en effet, à partir de la seconde moitié du xIV siècle, une multiplication des images établissant un lien entre l'évocation de la mort individuelle et l'invocation de la Trinité. Une miniature des Petites Heures, peinte vers 1390, montre le duc de Berry agenouillé face à une Trinité du type Trône de grâce; elle est accompagnée d'un texte en français qui doit être mis dans la bouche du duc, et où celui-ci prie Dieu-Trinité pour lui-même, à l'heure de sa propre mort ${ }^{5}$. 
Les images médiévales comportant un tel lien sont nombreuses et forment un réseau dont la complexité explique peut-être qu'il n'ait pas encore été scruté pour lui-même. Peut-être même n'a-t-il pas été seulement repéré en tant que tel. Je me propose donc, dans ce qui suit, de livrer, non pas une étude achevée, mais un premier lot d'observations. Comme la diffusion de ces images est d'ampleur européenne, recouvre plusieurs genres d'art et plusieurs types d'objet, et fait appel à plusieurs types

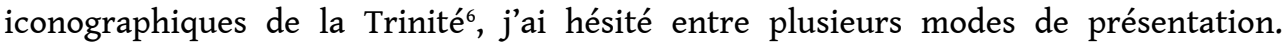
Réflexion faite, il m'a paru plus parlant, pour un premier repérage, de classer les images par genres d'objet, et de m'en tenir à trois d'entre eux : sépultures (I), épitaphes (II) et images de la bonne mort ou du jugement particulier (III). Après avoir donné quelques exemples parmi les plus anciens de chacun de ces groupes d'images, je risquerai pour finir quelques réflexions touchant à la genèse et à la signification de ce qui apparaît comme un phénomène historique de grande ampleur (IV). Mais ces dernières, j'en ai bien conscience, ne sont encore que de simples hypothèses de recherche à confirmer et à préciser par des travaux ultérieurs.

\section{Reliefs funéraires et pierres tombales}

4 L'un des plus anciens monuments funéraires à motif trinitaire ${ }^{7}$ est un grand relief en grès $(214 \times 123 \mathrm{~cm})$ daté du milieu du XIV siècle, aujourd'hui au musée de Mayence (fig. 1) ; jusqu'au xix ${ }^{e}$ siècle, il se trouvait dans la chapelle de l'hôpital du Saint-Esprit de Würzburg fondé en 1319 par Johann von Steren : deux autres donateurs de la famille Steren, sans doute Erko ( $† 1343)$ et sa femme, sont agenouillés à côté de leurs armoiries (un bouc), sous un Trône de grâce ${ }^{8}$. D'après certains chercheurs, cette œuvre aurait été créée pour remercier de la fin de la peste, et prémunir contre son éventuel retour - le motif de la Trinité, notamment ce type iconographique, a eu un temps, en effet, valeur apotropaïque : il suffisait de regarder vers la Trinité, croyait-on, pour être à l'abri de la peste $^{9}$. 
Fig. 1. Relief funéraire de la famille von Steren, $m$. XIV $s$.

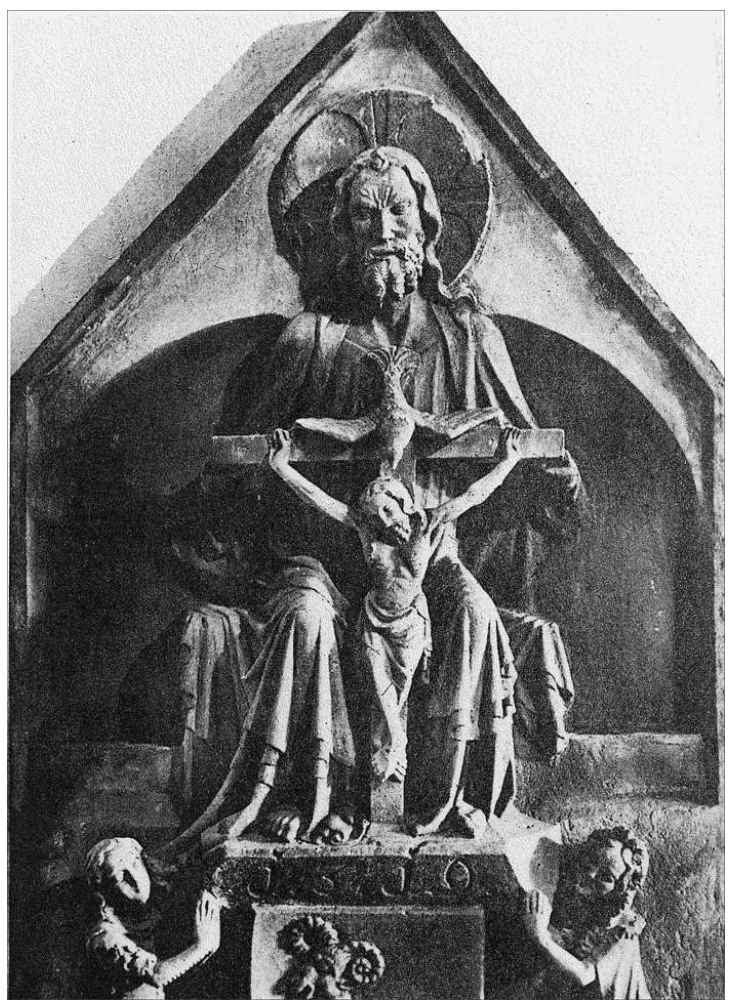

Würzburg, Mainfränkisches Museum.

5 Le relief funéraire de Jean Mariscault (ou Marescault), curé de Mainvault, daté "vers 1419 », aujourd'hui au musée des Arts décoratifs de Bruxelles, provient de Tournai : de manière inhabituelle, Dieu le Père, debout, est représenté avec la tête voilée de son manteau ${ }^{10}$. De même provenance, la plaque funéraire («lame») de Jean de Lours à Saint-Brice de Tournai ( Chi dsoubz cette lame, dit l'inscription, gist Jehans de Dours ni du manage $»^{11}$ ), de 1438, montre le défunt agenouillé sur la gauche, devant ses deux fils; il est présenté à Dieu par son saint Patron, Jean-Baptiste ; de l'autre côté du trône divin, sa femme Catherine suivie de ses quatre filles, sous la protection de sainte Catherine d'Alexandrie ; les écussons des deux familles sont représentés dans les angles supérieurs.

6 En 1429, Louis le Barbu (Louis VII de Bavière-Ingolstadt, 1413-1443), frère d'Isabeau de Bavière, l'épouse de Charles VI, commanda un projet de relief funéraire (un couvercle de sarcophage) à Hans Multscher (1400-1467) d'Ulm, un des meilleurs artistes de

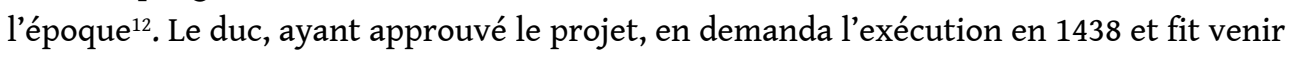
à cette fin, d'Ingolstadt, une grande plaque de marbre rouge. Ce projet ne fut jamais réalisé, mais le modèle en calcaire $(85 \times 31 \mathrm{~cm})$, lui, nous est parvenu (fig. 2). Le testament du duc, d'où proviennent ces informations, exprimait sa volonté bien arrêtée d'y voir figurer la Trinité, d'y être lui-même représenté agenouillé devant elle sur un ou deux genoux, "comme il semblera le mieux à l'artiste", et d'y voir portée une inscription avec la prière suivante : « $O$ sancta Trinitas miserere mei [...] et pardonne tous mes péchés [ces derniers mots en allemand]». Les figures se détachent sur un fond timbré d'un motif de soleil couronné à rayons. Agenouillé tout en bas sur le lion traditionnel, devant son écusson, le duc est en armure, coiffé, et tient une lance ; la tête légèrement rejetée en arrière, il fixe des yeux la Trinité, qui est figurée en Trône de 
grâce et entourée de trois anges, dont un qui paraît apporter la prière du duc au pied de la Croix. L'œuvre a su associer la crainte du Très Haut et la confiance en lui ${ }^{13}$.

Fig. 2. Louis le Barbu priant la Trinité, projet de relief funéraire, par Hans Multscher, 1435

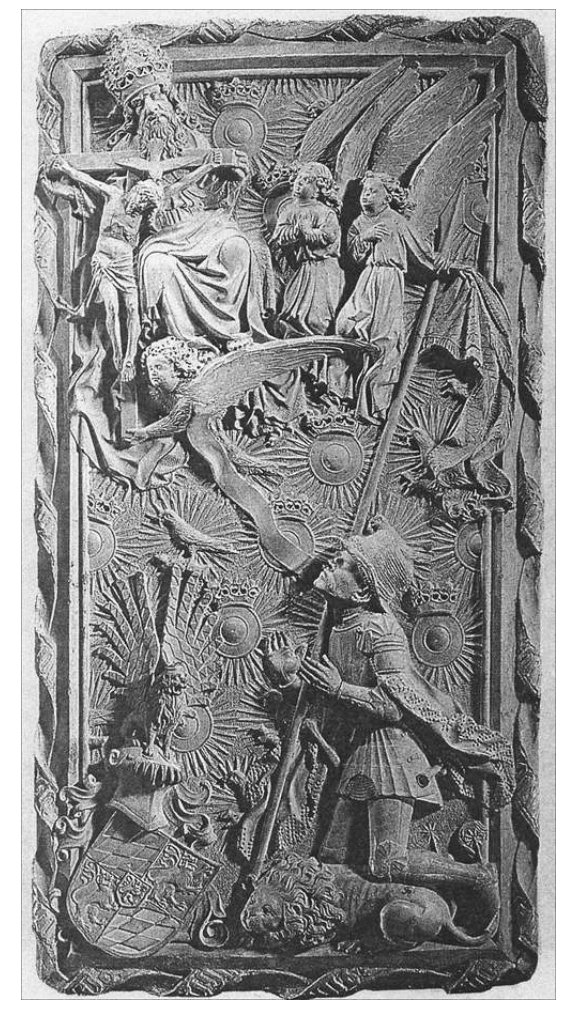

Munich, Bayerische Nationalbibliothek.

7 Plusieurs exemples anglais ou irlandais un peu plus tardifs peuvent être mentionnés, qui témoignent de l'extension européenne du phénomène à la fin du $\mathrm{Xv}^{\mathrm{e}}$ siècle. Un Trône de grâce sculpté, entre saint Édouard le confesseur et saint Patrick, orne l'un des grands côtés d'un sarcophage de la Christ Church de Waterford, en Irlande («vers $1482 »)^{14}$. Un autre relief funéraire de même type («vers $\left.1520 »\right)$ se trouve sur une cuve de St. Werburgh's à Dublin ${ }^{15}$. Une plaque en laiton (Brass) (1528) de la cathédrale SaintPatrick à Dublin (fig. 3) représente un clerc, Robert Sutton, en habit de cérémonie, agenouillé devant un motif de Trône de grâce d'où toute figure anthropomorphe est absente : ne reste que la forme générale du groupe, avec le bras horizontal de la croix et l'auréole crucifère du Père; le Trône de grâce, à cette date déjà, n'était plus jugé tolérable en Angleterre. Une banderole s'échappe des mains jointes de l'orant: In te domine speravi, non confundar in ceternum (c'est la fin de la prière du Te Deum). Les initiales $\mathrm{R}$ et $\mathrm{S}$ enlacées du donateur ont été trois fois reproduites, deux fois en bas et la troisième en surimposition sur le trône de Dieu: on peut y voir l'expression d'une espérance, celle d'être un jour pris en Dieu. Le texte latin souscrit, sur huit lignes, demande expressément que l'on prie pour Maître Robert, qui fut doyen de la cathédrale (Orate pro anima magistri Roberti Sutton). 
Fig. 3. Plaque funéraire de Robert Sutton, 1528

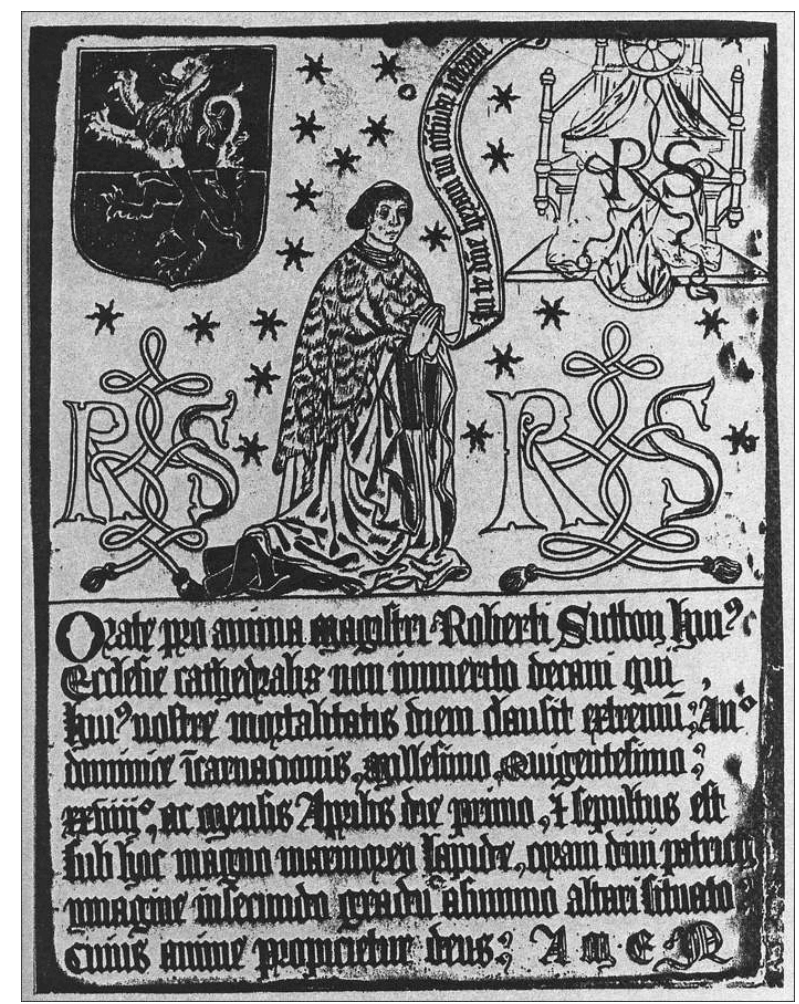

Dublin, cathédrale Saint-Patrick.

\section{Épitaphes}

8 Apparues seulement au $\mathrm{XIV}^{\mathrm{e}}$ siècle $^{16}$, les épitaphes peintes ou sculptées ont joui rapidement d'une grande vogue un peu partout en Europe, mais surtout en Europe du Nord, notamment dans les Pays-bas et tous les pays germaniques : il en reste plus de quatre cents dans la seule cathédrale d'Augsbourg ${ }^{17}$.

9 Au-delà de son sens traditionnel depuis l'Antiquité, celui de poème louant un défunt, rappelons que l'« épitaphe » désigne, chez les historiens de la période médiévale, une plaque commémorative distincte de la pierre tombale ${ }^{18}$, du sarcophage lui-même, ou de la tombe murale encastrée dans le mur et disposée verticalement ${ }^{19}$. L'épitaphe était destinée en général à être scellée dans un mur d'église. Elle pouvait aussi être fixée au tombeau ${ }^{20}$, mais elle était le plus souvent placée à distance de celui-ci, voire à distance $\mathrm{du}$ lieu d'inhumation proprement dit - un même défunt pouvait fort bien en faire disposer plusieurs.

10 L'épitaphe pouvait être de divers matériaux (plaque de pierre en grès ou en marbre, plaque de fonte ou de laiton, panneau de bois), avoir un format variable, comporter des figures et des éléments décoratifs plus ou moins riches et nombreux. Mais l'élément invariable restait l'inscription funéraire, qui comprenait en général deux parties : un court rappel de l'identité du défunt, avec au moins son nom et sa date de décès, et une prière plus ou moins développée : soit une prière adressée à Dieu ou à un saint, soit une interpellation $\mathrm{du}$ fidèle susceptible de déchiffrer l'épitaphe en passant devant, soit encore une prière dirigée vers ces deux types de destinataires, afin que les uns et les 
autres, par leurs prières (leurs "suffrages »), intercèdent auprès de Dieu, accordent ou obtiennent de lui le salut éternel du défunt. Globalement, la fonction de l'objet était triple : garder la mémoire des défunts, solliciter des prières pour son salut, et rappeler la vanité de ce monde ${ }^{21}$. Mais son but premier était sans doute d'obtenir l'intercession en faveur du défunt. Elle répondait donc pour l'essentiel à un besoin de sécurité post mortem et à un besoin corrélatif de sentiment de protection et de paix dès cette vie.

Dans les épitaphes à motif figuratif (les Allemands parlent d'épitaphe imagé, ou Bildepitaph) de la fin du Moyen Âge, l'on trouve représentés, outre le donateur, parfois couché lors de ses propres funérailles ${ }^{22}$ mais figuré vivant le plus souvent, agenouillé seul ou avec sa parentèle ${ }^{23}$, divers saints personnages de la foi chrétienne : le Christ (en Ecce Homo, homme de douleurs ${ }^{24}$, Véronique ${ }^{25}$, Christ de Pitié $^{26}$, Crucifiéé ${ }^{27}$, ou Jésus en prière au jardin des Oliviers ${ }^{28}$, ou Christ du Jugement dernier ${ }^{29}$ ), et/ou la Vierge Marie (Vierge à l'Enfant ${ }^{30}$ ou Nativitée $^{31}$, plus rarement un Couronnement de la Vierge), et/ou certains saints. Cette répartition des motifs ne recouvre pas du tout celle des sexes : il est frappant de constater, par exemple, qu'il y a une majorité d'hommes parmi les figures de donateurs agenouillés devant la Vierge.

Fig. 4. Épitaphe de Friedrich Mengot, peinture sur bois, v. 1370

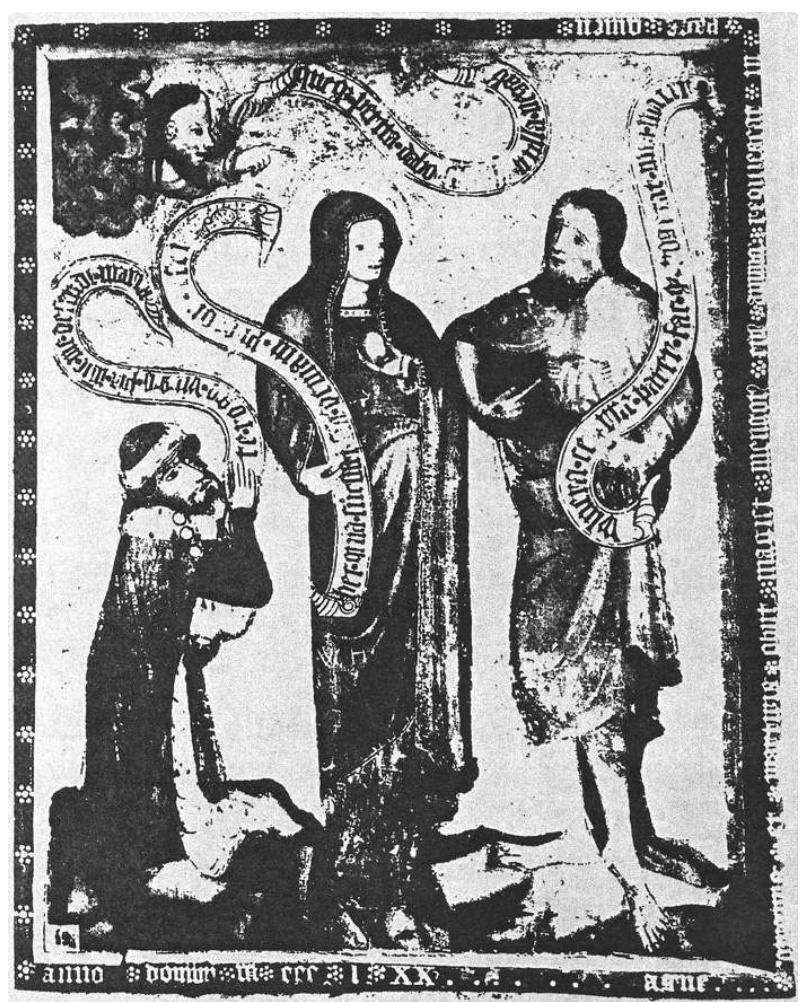

Heilsbronn, Protestantische Kirchenstiftung

12 Le premier Bildepitaph trinitaire est peut-être le mémorial de Frédéric Mengot ( $† 1370)$, médecin de Frédéric $\mathrm{V}$ de Nuremberg et des moines de Heilsbronn, conservé à la Protestantische Kirchenstiftung de Heilsbronn (fig. 4). Il s'agit d'un panneau de bois peint $(93 \times 71 \mathrm{~cm})$ que l'on peut, d'après certains spécialistes ${ }^{32}$, qualifier d'épitaphe, dans la mesure où il porte dans le cadre la date de décès du médecin, le jour de la Sainte-Agnès 1370 (21 janvier), suivie de son nom et d'une courte prière : anno domini m CCC LXX in die agne (tis)/ virginis et martiris obiit magister mengotus cujus anima 
requiescat in pace, Amen; Mengot figure sur la gauche, agenouillé, coiffé et revêtu d'un manteau à revers d'hermine; les textes des banderoles, rédigés en latin, donnent le contenu de cette intercession étagée ${ }^{33}$, qui passe par Marie puis par le Christ et aboutit à Dieu le Père, lequel apparaît dans le coin supérieur gauche du panneau. Le médecin supplie la Vierge: "Je te prie, Vierge miséricordieuse, défends-moi maintenant, Marie !»; celle-ci est à demi tournée vers le Christ et le prie en lui montrant un sein : «Comme tu as sucé ce sein, mon Fils, j'implore le pardon pour cet homme!»; le Christ montre sa plaie au côté à Dieu le Père: "Regarde les blessures, Père, et fais ce que demande ma Mère »; la réponse du Père ne se fait pas attendre: «tout ce que tu réclames, Fils, je te l'accorderai, et ne te refuserai rien $^{34}{ } »$. S'étant ainsi placé sous la protection d'aussi puissants intercesseurs que la Vierge et le Christ, le commanditaire se sera sans doute réconforté lui-même par l'assurance d'un verdict positif de la part de Dieu le Père.

Les autres exemples connus ne sont pas antérieurs aux années 1400. Provenant d'Italie, un retable d'attribution discutée qui fut naguère sur l'autel de la cathédrale de Florence et se trouve aujourd'hui aux Cloisters, v. 1402 (fig. 5) ; c'est le plus ancien tableau italien sur toile qui ait été conservé; il a été créé pour la chapelle funéraire de la famille Pecori, laquelle est représentée à genoux, à petite échelle, devant la Vierge qui la présente de la main; comme dans l'épitaphe Mengot, Marie s'adresse (en italien, évidemment) au Christ («Mon très cher Fils, par le lait que je t'ai donné, aie miséricorde de ceux-ci »), qui s'adresse à Dieu le Père («Mon Père, fais que soient sauvés ceux pour lesquels tu as voulu que je souffre la Passion $\left.{ }^{35} »\right)$; le schéma de composition est beaucoup plus majestueux, le Père placé en haut au centre domine l'ensemble; il ne répond d'ailleurs que par un geste bienveillant de la main; de ses doigts écartés émanent des rayons sur lesquels paraît glisser la Colombe piquant vers le Christ $^{36}$.

Fig. 5. Épitaphe de la famille Pecori, peinture sur toile, v. 1402 


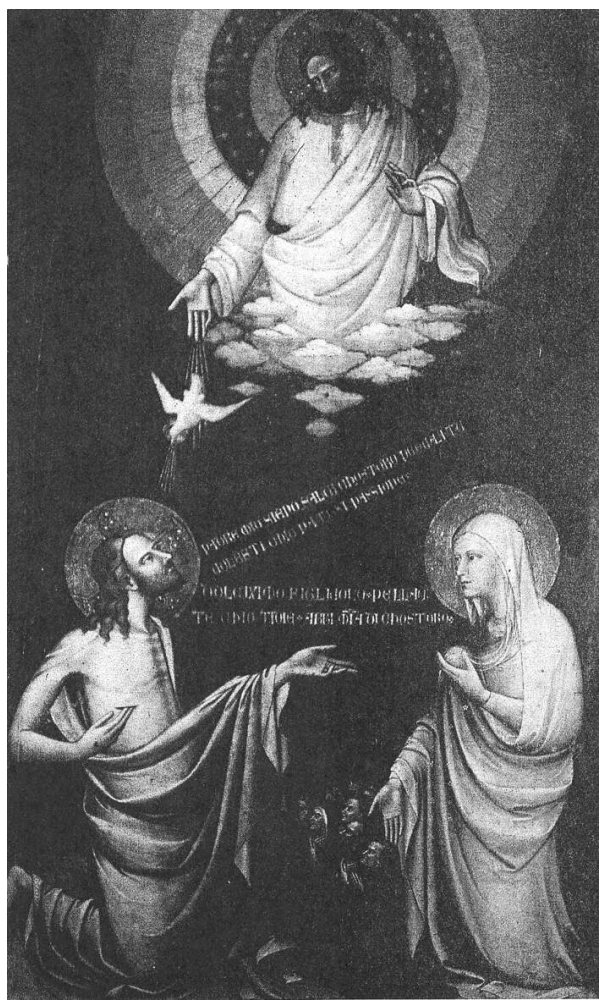

New York, Cloisters.

À partir de la deuxième décennie du siècle, les épitaphes à motif trinitaire se multiplient, notamment en Flandre, Angleterre et Allemagne, et, dans une moindre mesure en Italie - la fameuse fresque de la Trinité peinte par Masaccio à Sainte-MarieNouvelle de Florence (vers 1428$)^{37}$ comporte au registre inférieur, sous deux priants agenouillés, un transi.Je me borne ici à fournir quelques indications sur celles de Flandre.

15 Au début du Xve siècle, dans les Pays-Bas méridionaux, le Trône de grâce au pied duquel sont agenouillés des hommes et des femmes en prière devient l'un des thèmes privilégiés de l'art funéraire ${ }^{38}$. Certaines épitaphes ont été détruites, en particulier en 1566, par les iconoclastes se réclamant des idées de la Réforme ${ }^{39}$. Beaucoup subsistent néanmoins, dont le plus grand nombre provient du Hainaut, et particulièrement de Tournai ${ }^{40}$. Elles représentent ou bien le donateur seul (notamment quand il s'agit d'un ecclésiastique) ou bien un couple de donateurs agenouillés devant le groupe trinitaire (en général avec la "protection » de leurs saints patrons), le donateur étant placé éventuellement en face de sa femme ou de ses femmes successives (en cas de remariage), et les enfants du couple se trouvant rangés d'un côté ou de l'autre, selon leur sexe. "Cette iconographie révèle le développement d'un sentiment déjà moderne de la famille dans certains milieux de la nouvelle société de l'Ancien Régime ${ }^{41}$. »

16 À Sainte-Waudru de Mons, se trouvent l'épitaphe sculptée de Guillaume de Peissant ( $†$ 1409) et de son épouse Agnès de la Roque, figurés à genoux mains jointes devant un Trône de grâce, avec leurs " patrons » respectifs, les saintes Barbe et Agnès ${ }^{42}$, et celle de Guillaume de Bruxelles ( $† 1430)$ et Jeanne Nokarde ( $† 1437)$ : les deux époux sont agenouillés avec leurs blasons respectifs sous trois pinacles de facture gothique devant un Trône de grâce juché sur un petit piédestal; le fils, Nicolas, une fois n'est pas 
coutume, est placé derrière la mère, à côté des trois enfants dans le saloir qui sont l'attribut de son saint patron ${ }^{43}$. Même distribution des attitudes et des motifs dans l'épitaphe du chanoine Robert de Quinghien $(\dagger 1429)^{44}$ conservée au musée d'Histoire et d'Archéologie de Tournai : pas de femme, cette fois, et pour cause ; Robert, chanoine d'Antoing, est présenté par saint Jean-Baptiste, il est agenouillé devant un Trône de grâce désaxé (exactement comme dans les Petites Heures), avec le Père siégeant devant une tenture tenue par des anges ${ }^{45}$.

17 À la cathédrale de Tournai, est conservé le relief épitaphe de Tasse Saveris (= Eustache Savary), daté de 1426 : agenouillés tout contre le Trône de grâce et placés eux aussi sous des baldaquins gothiques polygonaux, les donateurs, accompagnés de leurs saints patrons (à gauche, le donateur et son fils; à droite, l'épouse, Catine de la Walle, assistée de sainte Catherine d'Alexandrie); une longue inscription a été gravée au registre inférieur ${ }^{46}$. Dans la même église, l'épitaphe du chanoine Jehan Lamelin $(\dagger 1470)$ : les têtes manquent toutes, mais l'on reconnaît le chanoine agenouillé devant la Croix, avec son saint patron qui lui a mis la main sur l'épaule ; en face, la Vierge Marie agenouillée elle aussi, et dévoilant son sein; dans le coin supérieur droit, Dieu le Père dans une gloire circulaire ${ }^{47}$. Cette dernière œuvre a été faite sur le même modèle qu'une miniature des Heures de Catherine de Clèves ${ }^{48}$. Toujours à Tournai, à l'école Saint-Luc cette fois, est conservé le monument funéraire d'Antoine Watiers († 1425) : la disposition de la famille (avec de nombreux enfants : cinq fils derrière le père, trois filles derrière les deux épouses successives, chacune avec sa patronne) est toujours la même ${ }^{49}$. Un exemple tout à fait typique des monuments de cette provenance est l'épitaphe d'Enguerrand de Wisquette (fig. 6), " après 1468 ", provenant de Saint-Maurice de Lille, aujourd'hui au musée des Beaux-Arts de cette ville ${ }^{50}$ : Enguerrand, en armure, est agenouillé à gauche, avec, debout derrière lui, saint Jean Baptiste qui lui a mis la main sur l'épaule pour le présenter à Dieu; sur la droite, ses deux femmes successives, présentées par sainte Catherine d'Alexandrie. 
Fig. 6. Épitaphe d'Enguerrand de Wisquette, relief en pierre, après 1468

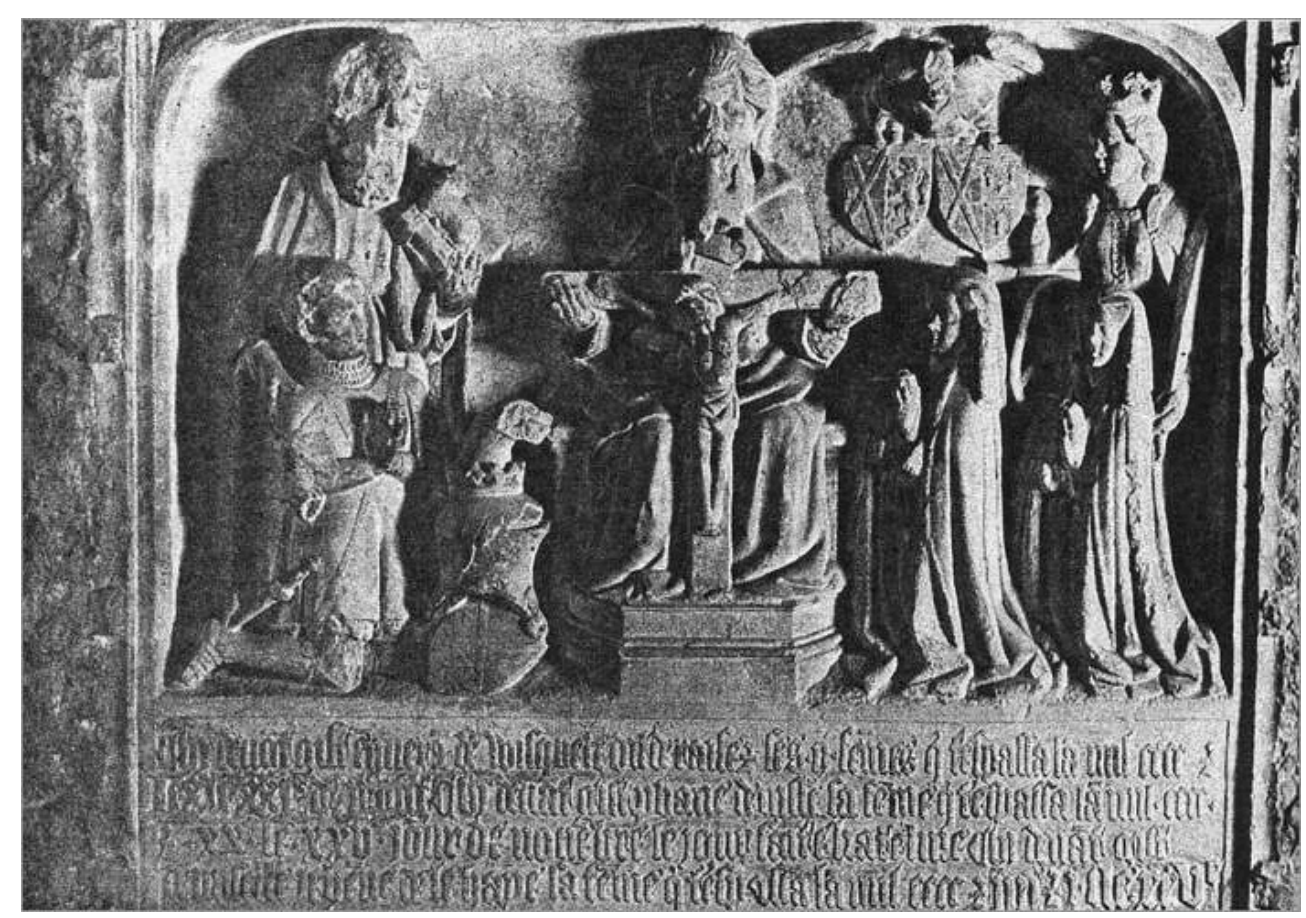

Lille, musée des Beaux-Arts.

\section{Images de l'homme sur son lit de mort}

Un troisième groupe de figurations associant la Trinité et la mort individuelle est constitué cette fois par des images de dévotion. On ne s'attardera pas, ici, sur les séries de gravures de l'Ars moriendi, qui ont leurs études de référence ${ }^{51}$. Contentons-nous de rappeler au passage que certaines d'entre elles font apparaître, avec éventuellement la Vierge et le saint patron, le groupe de la Trinité au chevet du mourant, de l'autre côté du lit par rapport aux démons ${ }^{52}$. La suite de cette section de notre recherche voudrait attirer plutôt l'attention sur les images où l'âme du mourant, représenté également couché sur son lit d'agonie, est l'objet d'une âpre négociation entre l'enfer et le ciel.

Dans un «psautier-livre d'heures » du nord de la France (Picardie), réalisé vers 1340, et conservé en Avignon (BM, ms 121, fol. 73v), en tête des prières pour les morts, se trouve une miniature en pleine page (fig.7), dans un cadre rectangulaire orné de motifs de vigne, qui présente une variante précoce des images de la Double Intercession ${ }^{53}$. Reliant les figures peintes sur fond or quadrillé en diagonale et piqué de petits motifs circulaires, huit banderoles, numérotées de I à VIII dans des cartouches à fond rouge ou bleu, ont un texte sur deux lignes, la dernière formant un bas de page, avec une texte sur quatre lignes. Ces numéros suggèrent à la fois le déroulement de la plaidoirie et l'ordre de lecture. 
Fig. 7. Image de la bonne mort, miniature, psautier livre d'heures [France du Nord], vers 1340

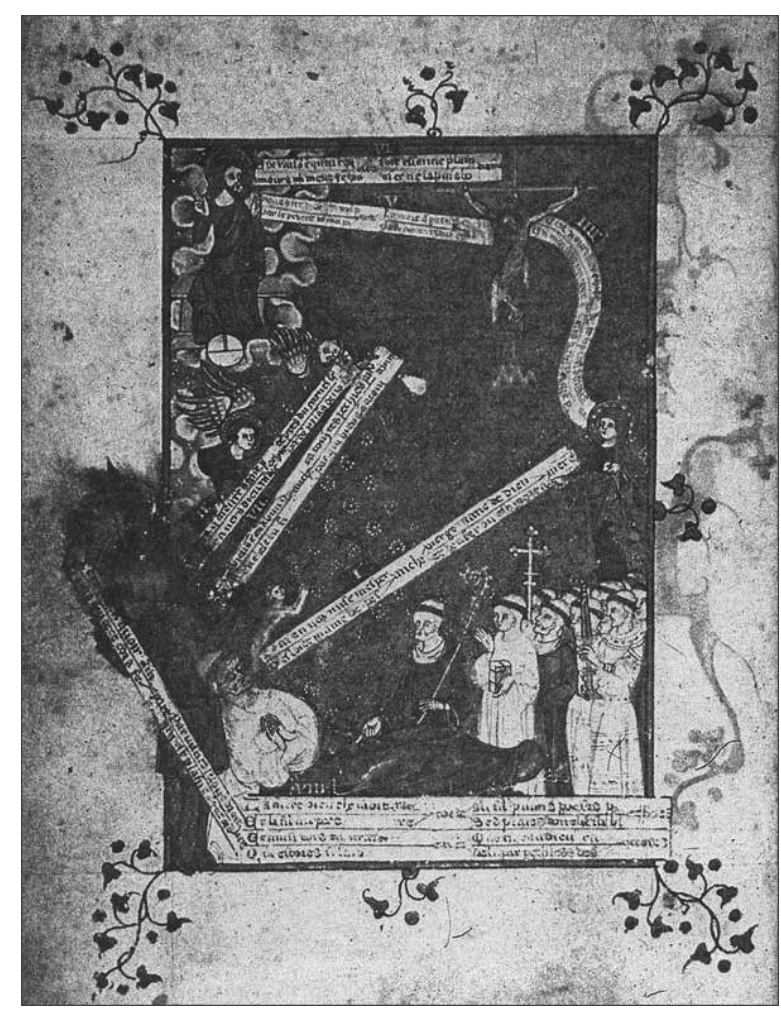

Avignon, Bibliothèque municipale, ms. 121, fol. $73^{\mathrm{v}}$.

La miniature fait voir, en bas, un religieux (portant une couronne de cheveux) sur son lit de mort, autour duquel sa communauté s'est rassemblée pour le dernier adieu ; elle fait procession vers le lit, abbé en tête (avec collier de barbe blanche) tenant la crosse, suivi de neuf religieux, dont quatre portant respectivement un livre (il est revêtu d'une coule blanche), une croix de procession, et deux, vêtus d'une aube ornée, qui tiennent un cierge. Dans la mesure où un psautier livre d'heures est normalement destiné à une personne laïque, plutôt que de voir une contradiction entre cette destination et cette mise en scène, on est invité à lire cette dernière comme le modèle de la bonne mort. Le mourant est à la fois mort en vivant : il rend l'âme, mais il parle (du moins lui prête-ton des ultima verba de circonstance). Il est allongé sur son lit, les yeux clos, et son âme sort de sa bouche sous la figure conventionnelle d'un enfant nu ; mains jointes, il prie la Vierge, et la banderole qui porte le texte de sa prière fait comme un pont entre lui et elle; celle-ci apparaît en pied, vêtue d'une robe bleue, dotée d'un voile orange et nimbée (son nimbe est rouge et piqué de points blancs), au-dessus du cortège : « Je ai en vos mise m'esperanche, Vierge Marie de Dieu mère: Desloiet m'ame de pesanche, Et d'Enfer où est mort amère " (I); Satan, au corps brun, vêtu seulement d'un pagne orange, de stature imposante, se tient derrière lui : «Je requiers avoir à me part, Par Justice selon droiture, l'ame qui de ce cors se part, Qui est plaine de grant ordure » (II) ; la banderole d'un premier ange (un ange de justice ?) est à lire ici (III); la Vierge intercède pour le religieux, qu'elle désigne de la droite, auprès de son Fils représenté en croix (IIII ; le texte de sa prière est en rouge : "Ceir nourit xx chier... », appuyant sa plaidoirie du geste de montrer l'un de ses seins de la main gauche; le Christ à son tour montre ses plaies à son Père (un Dieu-Christ au nimbe uni, avec tunique rouge sur robe 
orange, la tête tournée vers son Fils, bénissant et siégeant sur un trône banc à l'intérieur d'un ourlet de ciel conventionnel) en lui transmettant la prière de sa Mère (V); Dieu accorde : «Six raisons est que ta requeste Soit essaucie plainement: Amour m'y meut qui est honneste, Nier ne le puis bonement » (VI) ; le second ange (un ange de miséricorde?), qui descend directement du trône de Dieu, promulgue la sentence divine (VII) ; la dernière banderole redonne la parole au mourant, pour une action de grâce. Des anges commentent la scène et le clergé en tire les conclusions. La figure du démon a été effacée par un lecteur, comme il arrive souvent pour les personnages fâcheux (son image, à elle seule, portait malheur) ${ }^{54}$.

21 Autre image de ce type de procès, la grande miniature $(36 \times 24 \mathrm{~cm})$ (fig. 8) d'un manuscrit du Fons Virtutum provenant de Nuremberg, vers 1400, et conservée à Florence ${ }^{55}$. Les banderoles sont garnies de textes en vers latins (le nombre de pieds est irrégulier). En bas, rendant l'âme, le mourant dans son lit, les bras croisés dépassant audessus du drap (O Spes in morte/mihi parce, Maria precor te) ; au pied du lit, sur la droite, la noire silhouette de Satan (Hanc animam posco/Quia plenam crimine nosco); au-dessus de lui, cette fois rapprochés, en face l'un de l'autre, la Vierge à gauche (Hanc quia suxisti/Fili veniam precor isti) et le Christ à droite (Vulnera cerne pater/Fac quod rogavit mea mater), plaidant chacun avec ses propres gestes; derrière la Vierge, une fois n'est pas coutume, saint Jean (Hic si peccavit/Nece pressus opere rogavit); au-dessus encore, dans une mandorle, Dieu le Père (figuré en vieillard) répondant à son Fils (Nate petita dabo/quae petis nulla negabo); dans l'angle supérieur gauche, un ange (Ecce fons virtutum/Viciis jubet esse solutum $)^{56}$.

Fig. 8. Image de la bonne mort, miniature, Fons virtutum [Nuremberg], v. 1400

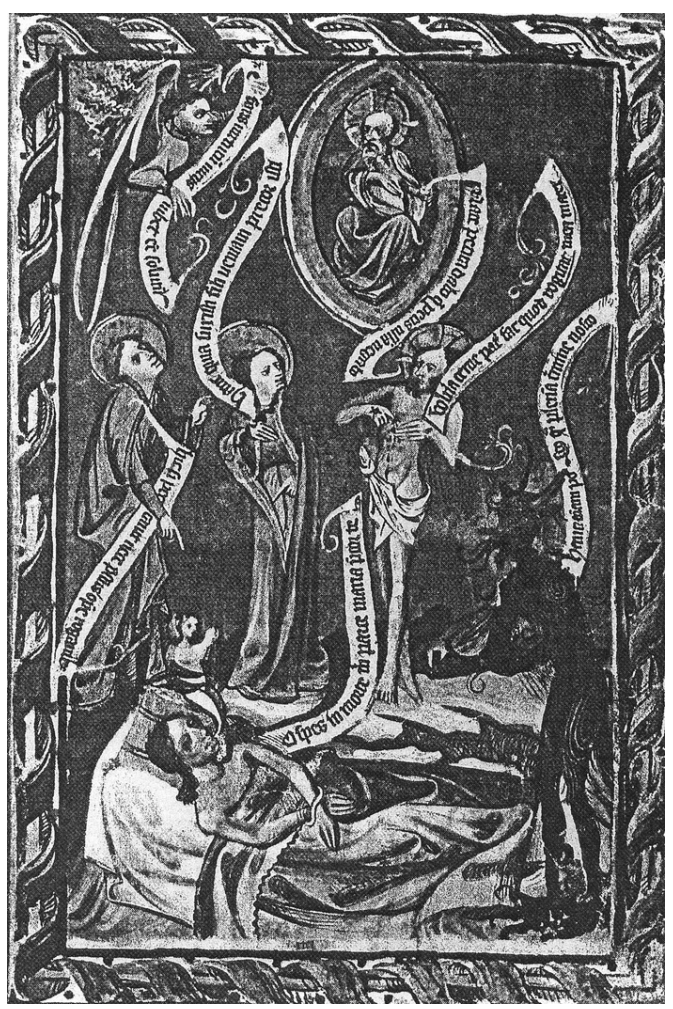

Florence, Biblioteca Nazionale, Codex B.R. 38. 
Le lien entre cette imagerie et la liturgie des défunts est de nouveau explicite dans une miniature de missel conservé à Cracovie ${ }^{57}$, ou le même scénario réapparait, il est vrai sous forme simplifiée (fig. 9), dans l'initiale R de l'antienne Requiem aeternam dona eis domine, pour la célébration d'un enterrement (In die depositionis unii defuncti): le mourant est dans son lit, les yeux fermés, au pied de la croix, son âme s'échappe, que le diable réclame comme son bien (hanc animam posco quam plenam criminis nosco); mais la prière du mourant monte vers Marie (Sancta Maria), laquelle s'adresse au Christ (Natus ubera succisti Fili veniam precor isti), qui se tourne alors vers le Père (fac quod rogat mea mater), qui lui répond de manière positive (Fili petita dabo, quae tu petis non denegabo), ce qui donne à un ange de conclure (et fons virututm iubeat eam esse solutam) : l'affaire se conclut par un happy end prometteur et rassurant.

Fig. 9. Image de la bonne mort, miniature, missel, vers 1450

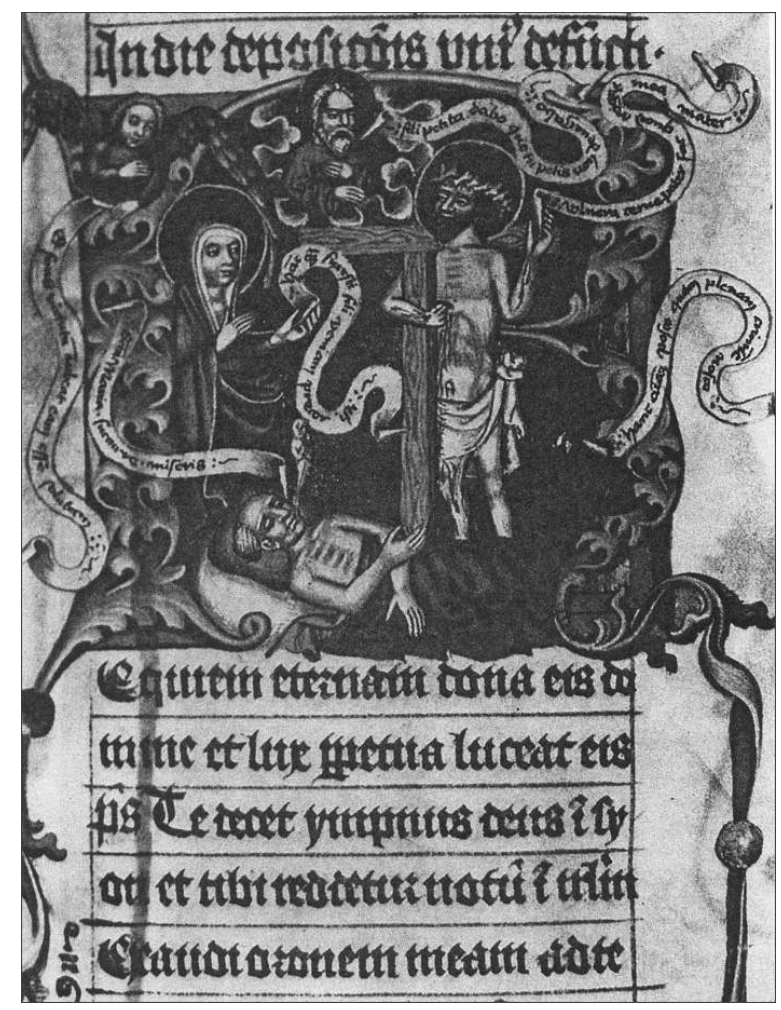

Cracovie, Archiwum i Biblioteka Kapituly Metropolitalnej na Wawelu, ms n² KP, fol. CCCXCVIII

Cette troisième famille d'images peut être suivie jusqu'au $\mathrm{XVII}^{\mathrm{e}}$ siècle ${ }^{58}$. Contentonsnous, dans le cadre de cette recherche, qui s'arrête au début $\mathrm{du} \mathrm{XvI}^{\mathrm{e}}$, de présenter la version qu'en a donné Lucas Cranach l'Ancien (1472-1553), en 1518, dans un tableau (fig. 10) intitulé Der Sterbende («Le mourant»), conservé à Leipzig, qui récapitule le rapport que la catéchèse et la dévotion de la fin du Moyen Âge ont établi entre la mort individuelle et la Trinité rédemptrice. 
Fig. 10. Der Sterbende, panneau peint par Lucas Cranach l'A., 1518

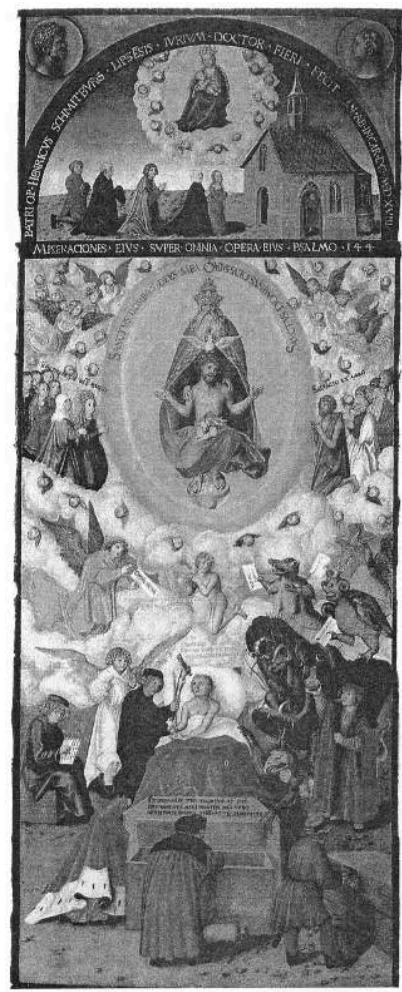

Leipzig, Museum der bildenden Künste.

Ce tableau est l'exemple même de la première manière du peintre, qui traitait des thèmes usuels, à la manière de l'Ars moriendi $i^{59}$. Il est construit en hauteur $(93 \times 36 \mathrm{~cm})$, sur trois registres. Tout en bas, un homme sur son lit de mort, entouré de sa parentèle ; un coffre vide est au pied du lit, tandis qu'un homme, sur la droite, parait fermer un cercueil; le mourant se tourne vers un ministre qui lui tend un crucifix; derrière le ministre, un ange, l'ange gardien probablement; sur la gauche, un homme en train d'écrire (le notaire, prenant le testament sous la dictée) ; l'épouse se tient à genoux ; sur la droite, un médecin examinant les urines, et des monstres prêts à avaler l'âme du défunt; celle-ci est figurée nue, comme il est traditionnel, s'élevant au-dessus du mourant, entre un ange portant un billet (opera bona) et un premier démon portant un autre billet, qui en précède deux autres également porteurs de " cédules ", ces billets valant créance pour les dettes contractées envers le monde des ténèbres; l'âme est évidemment réclamée par les deux parties, au cours d'une plaidoirie contradictoire ; au registre intermédiaire, entourée d'anges et de saints à genoux intercédant, une Trinité du type Compassion du Père : le Fils en Christ de Pitié descendu de Croix est assis, les pieds sur le globe du monde et l'Agneau pascal sur les genoux, tout contre devant le Père revêtu d'un grand manteau et coiffé de la tiare triple; dans le bord supérieur de la mandorle, on peut lire deux fois l'inscription en capitales: Sanctus Dominus Deus Sabaoth; au registre supérieur, dans un compartiment en demi-cercle formant le couronnement du panneau, une famille (la fille, sa mère, puis le père entre ses deux fils) agenouillée devant une église, avec une Vierge à l'Enfant donnant le sein et apparaissant dans le ciel environnée de têtes d'ange; une inscription latine se développe dans le cadre : sur la partie horizontale : Miseraciones eius super omnia opera eius, Psalm 144; sur le bord semi circulaire : Patri Optimo Henricus Schmitburg Lipsensis 
Iurium Doctor fieri fecit Anno ab Incarnatione MDXVIII ("au meilleur père, Henri Schmitburg de Leipzig, docteur en droit, fit faire [s.e. cette image] l'année 1518 après l'Incarnation »). Ainsi, ce tableau procède de l'amour filial, et l'on doit donc supposer qu'il était fait pour créer une impression rassurante: mais le sentiment de sécurité qu'on a pu en tirer n'a pas d'autre source que la promesse de salut offerte par la présence dans le sein du Père de l'Homme de douleurs, et les prières des siens ${ }^{60}$.

\section{Genèse et signification d'un phénomène}

Le rapide panorama que nous avons dressé est évidemment incomplet. Manquent à l'appel non seulement bon nombre d'images relevant des trois catégories d'objet présentées, mais aussi certains tableaux relevant d'autres genre encore. Ainsi de l'In memoriam de Jacopo de Sellaio du musée de Tokyo, peint en 1479-1480 pour une famille florentine (la peste sévit de nouveau à Florence de 1479 à 1483) ${ }^{61}$, et faisant office d'exvoto (fig. 11) : juché dans une mandorle lumineuse sur fond de ciel sombre, Dieu le Père soutient l'axe horizontal d'une haute croix au pied de laquelle ont été placés à même le sol deux défunts, une mère et son enfant, victimes de l'épidémie ; ils sont enveloppés dans leur linceul (seuls les visages sont visibles), entourés de pleurants, et montrés à Dieu par la Vierge et saint Jean; au fond, la ville de Florence; sur deux rochers, à gauche, le Sacrifice d'Abraham et, à droite, le Don de la Loi au Sinaï.

Fig. 11. In Memoriam, panneau peint par Jacopo de Sellaio, v. 1480

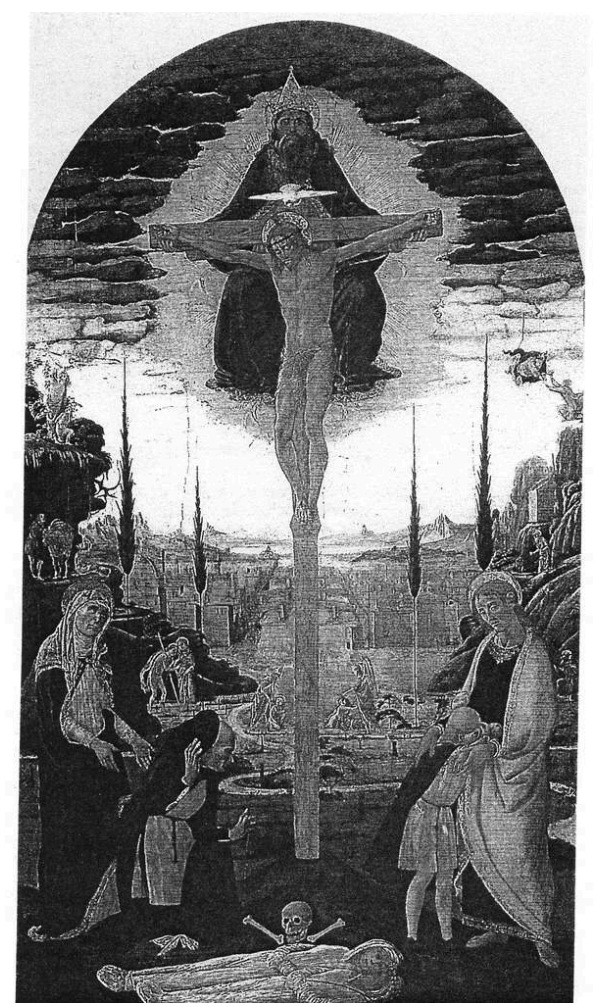

Tokyo, musée des Beaux-Arts.

Mais nous en avons sans doute assez dit pour suggérer que l'association des deux thèmes de la mort individuelle et de la Trinité dans un même objet peint ou sculpté paraît caractériser l'art et la spiritualité de la fin du Moyen Âge et constituer un 
épisode singulier de l'histoire des mentalités religieuses d'Occident. Cette association ne semble en effet connaître dans l'histoire du christianisme latin ni antécédent ni conséquent. Elle est rarissime dans l'art chrétien avant le $\mathrm{XIV}^{\mathrm{e}}$ siècle. Durant tout le $\mathrm{I}^{\mathrm{er}}$ millénaire, le panorama des images de la Trinité, encore très réduit, ne comporte, sauf erreur, aucun rapport explicite à la mort individuelle, ni à la prière pour les défunts ${ }^{62}$, même s'il se peut que le placement des défunts sous la protection de la Trinité n'ait pas été complètement étranger à l'art funéraire des premiers siècles ${ }^{63} . \mathrm{Si}$ l'on regarde maintenant en aval, cet autre constat s'impose : à partir du XVII siècle, les images trinitaires de la bonne mort ne survivent guère que dans le domaine de l'imagerie, et le thème trinitaire se raréfie petit à petit dans les tombeaux et épitaphes ${ }^{64}$. Et au total, la Trinité, en tant que motif, est quasi absente de l'art funéraire depuis le XVIII ${ }^{e}$ siècle ${ }^{65}$ - seul le Trône de grâce se maintient et continue de faire des apparitions sporadiques en contexte funéraire, jusqu'au $\mathrm{xx}^{\mathrm{e}}$ siècle $^{66}$.

Pour rendre compte de la spécificité médiévale de ce phénomène, on peut d'abord songer à quelques considérations formelles touchant à ses conditions de possibilité, et alléguer ensuite certaines données historiques, liturgiques et théologiques dont la réunion constitue sinon une explication dudit phénomène, du moins un faisceau de facteurs susceptibles de l'éclairer.

À partir du XII ${ }^{e}$ siècle ou de la fin du XI ${ }^{e}$ siècle, avec l'apparition du type iconographique Trône de grâce, qui montre le Christ en croix entre les genoux de Dieu le Père, et surtout à partir du siècle suivant, qui est marqué par la diffusion rapide de ce type, les conditions paraissent réunies pour que l'association se fasse entre la pensée de la Trinité et la pensée de la mort personnelle et s'expose dans les arts figuratifs. Les nombreuses occurrences de priants agenouillés devant ce groupe trinitaire, dont les plus anciennes remontent au début $d u$ XIII $^{e}$ siècle ${ }^{67}$, auront préparé le Trône de grâce à sa mise au service de la prière pour les défunts. Quand se renforcera le thème majeur de l'imitation du Christ, et lorsque le rapport deviendra étroit, dans la spiritualité, entre la mort des fidèles et la mort de leur Seigneur sur la croix, l'évocation de la Trinité du type Trône de grâce en contexte funéraire deviendra presque logique si l'on tient encore compte de la multiplication, à cette époque précisément, des prières adressées directement à la Trinité, dont témoigne, entre autres, l'invocation à la Trinité placée en tête des litanies des saints.

Ces considérations générales, dira-t-on, ne sont pas dénuées de pertinence, mais n'expliquent pas assez, aux yeux de l'historien, pourquoi c'est précisément partir du $\mathrm{XIV}^{\mathrm{e}}$ siècle que les images de la Trinité en contexte funéraire se sont multipliées. Le faisceau de «raisons » auxquelles on peut ici faire appel, évidemment sous bénéfice d'inventaire, paraît le suivant : montée en puissance de la dévotion pour la Trinité (1), série de calamités publiques suggérant de recourir directement à Dieu et non plus seulement à ses saints (2), dévotion trinitaire de notables prestigieux, ayant valeur d'exemple (3) ; progressive intégration de la Trinité dans le système de l'intercession (4).

30 1/ Rappelons d'abord que la dévotion des fidèles envers la Trinité n'a cessé de se généraliser en Occident au cours des derniers siècles du Moyen Âge. La Trinité ne resta pas prisonnière des intérêts et curiosités théologiques des clercs ${ }^{68}$. Au contraire, on peut parler d'une "popularité » croissante de ce mystère à partir du XII ${ }^{e}$ siècle. En témoignent, entre autres indices, la place désormais accordée à la Trinité dans la liturgie, la catéchèse, la prédication des Mendiants, les livres d'heures destinés aux 
laïcs, les dédicaces d'églises, de couvents, chapelles et autels privés, la toponymie, l'iconographie funéraire, les formules testamentaires, les livres juratoires, les visions mystiques, les prières en langue vernaculaire, et de multiples usages plus ou moins superstitieux, liés à la sacralisation très répandue du nombre trois ${ }^{69}$, à commencer par celui du signe du croix. La liturgie, en particulier, a dû jouer un grand rôle : certaines prières à la Trinité font leur apparition dans le canon de la messe ; l'invocation de la Trinité et de chacune des trois Personnes est ajoutée en tête des litanies; apparue dès le début $\mathrm{du} \mathrm{x}^{\mathrm{e}}$ siècle, la fête de la Trinité se répand de proche en proche dans toute l'Europe du Nord, avant d'être finalement étendue à l'Église universelle, par Jean XXII, en 1334. L'apparition, dans ce contexte, de certains ordres religieux, tel celui fondé en 1198 par Jean de Matha et Felix de Valois, l'« ordre des Trinitaires et de la Rédemption des captifs ", est un symptôme parmi beaucoup d'autres.

L'apogée de cette faveur populaire, qui confinera parfois à la "familiarité excessive » (J. Huizinga), semble atteinte au cours $d u x^{e}$ siècle. L'iconographie constitue à cet égard un témoignage éloquent et une mine d'informations concernant ce chapitre de l'histoire de la spiritualité : les plaques funéraires en laiton (en Angleterre), les reliefs de clefs de voûte, les groupes sculptés, les retables, les reliefs et motifs des épitaphes nous renseignent et sont une attestation non équivoque de la faveur du dogme de la Trinité dans l'esprit des fidèles de toutes les classes de la société, dans la mesure où la commande de la plupart de ces objets et leur iconographie elle-même passaient par les laïcs et reflétaient leurs vœux explicites : la Trinité a nourri la prière, les multiples dévotions et les pratiques religieuses les plus variées d'un nombre grandissant de fidèles au fur et à mesure que s'écoulent les siècles du Moyen Âge.

2/ Il est permis de supposer que les calamités publiques de grande ampleur ont contribué à cette nouvelle orientation de la spiritualité. Se trouvant à Rome en 1349, et interrogée sur les remèdes à prendre, sainte Brigitte de Suède en suggéra trois : abolir les vanités vestimentaires, faire l'aumône aux nécessiteux, donner l'ordre aux prêtres de célébrer chaque mois une messe en l'honneur de la Trinité ${ }^{70}$. Toujours est-il que la Peste noire pourrait avoir joué un grand rôle dans la popularité du Trône de grâce. En Italie, par exemple, ce type iconographique apparaît avec une fréquence croissante dans les dix années qui suivent le déclenchement de la Peste. Il est souvent associé aux saints anti-pesteux ${ }^{71}$. De nombreux retables furent créés en ce sens, et aussi des gonfalons, ces bannières de procession transportées dans les rues pour combattre la peste, notamment en Toscane et en Ombrie ${ }^{72}$. Il est également possible que des images trinitaires déjà existantes aient alors été utilisées en ce sens. Ce recours à la compassion efficace du Dieu trinitaire n'a pas été le seul fait de l'Italie du Trecento. On en connaît de nombreuses attestations en Europe aux xIV et $\mathrm{Xv}^{\mathrm{e}}$ siècles.

Assurément, les fidèles comptaient sur la protection que pouvaient leur apporter les saints dits "antipesteux", tels les saints Roch, Christophe, Nicolas de Tolentino, Sébastien ${ }^{73}$ et Jean-Baptiste, plus ou moins spécialisés dans la protection des humains contre la peste, ou encore la Vierge au manteau, capable de dévier ou de briser les " flèches de peste » décochées contre les humains du haut du ciel par le Christ ou par Dieu le Père avec ou sans le concours d'anges. Mais le recours direct à la Trinité, en pareil cas, a sans doute été beaucoup pratiqué; compte tenu de l'ampleur de l'hécatombe, on a sans doute estimé qu'il était indiqué de s'adresser directement à Dieu plutôt qu'à ses saints. 
3/ L'exemple de la confiance envers la Trinité à l'heure de la mort semble être venu de haut, c'est-à-dire des clercs de haut rang et des princes, et s'être propagé à partir de là, de proche en proche, aux autres couches sociales, l'imitation de la noblesse, chez ceux que l'on appellera les bourgeois, et jusque dans le petit peuple, ayant servi de processus relais. Jean de Berry priant la Trinité de lui être favorable à l'heure de sa mort est un exemple parmi d'autre de ces dévotions nobiliaires qui semblent avoir fait école. Quelques années auparavant, le père du duc, Charles V, roi de France, avait décidé que son monument funéraire, à Saint-Denis, serait disposé sous une clef de voûte ornée d'un motif de la Trinité. Son frère, Philippe le Hardi, duc de Bourgogne, semble avoir partagé cette ferveur pour la Trinité, et pris des mesures pour sa propre chapelle funéraire à l'abbaye de Champmol, le Saint-Denis de la maison de Bourgogne, etc.

4/ Enfin, il semble que l'on puisse parler, à la veille des Réformes, de l'intégration progressive de la Trinité dans le système de l'intercession. La présence du Christ sur les monuments funéraires et images d'intercession, et notamment d'une figure du Christ de la Passion, coulait de source, pourrait-on dire : c'est dans les «mérites» de sa Passion que les fidèles se confient en définitive, et c'est sur son intercession de médiateur que se fonde, d'un point de vue scripturaire et théologique, tout le système de l'intercession de la Vierge et des saints. Comme Panofsky l'a bien souligné ${ }^{74}$, la présence sur les épitaphes de l'Homme de douleurs (ou de tout motif du Christ de la Passion) constituait sans doute un gage de salut : on considérait qu'il intercède auprès $\mathrm{du}$ Père usque ad eternum. La présence de la Vierge dans les images que nous avons décrites, pareillement, n'a pas de quoi surprendre. Certaines prières de la fin du Moyen Âge, tel le Salve regina, la présentent et la prient comme l'avocate par excellence ${ }^{75}$. La fin de la prière du «Je vous salue Marie ", à savoir « Priez pour nous, pauvres pécheurs, maintenant et à l'heure de notre mort ", commence d'être attestée à la fin du Moyen Âge ${ }^{76}$ et suppose la croyance que Marie, Mère de miséricorde, se montrera secourable envers les mourants, pourvu qu'on l'en prie ${ }^{77}$. De même, la présence de certains saints, en particulier du saint patron du défunt et plus généralement des « saints de la bonne mort ", est fréquente et bien repérée dans un certain nombre d'épitaphes; elle traduit elle aussi l'espoir de leur soutien et de leur intercession.

Moins banale, et peut-être aussi moins bien repérée, est la présence de la Trinité « tout entière ", pourrait-on dire, dans ces images de protection, faisant apparaître, au-delà des bénéficiaires et des divers avocats et acteurs de l'intercession, son ultime instance ou, si l'on préfère, son destinataire. Tout se passe comme si les fidèles de la fin du Moyen Âge avaient tenu à visualiser le processus de leur propre passage en jugement après la mort, et à manifester toute l'espérance qu'ils mettaient, en vue de leur salut éternel, dans les intercesseurs les plus efficaces qui se puissent concevoir, à savoir le Christ et la Vierge.

37 Cette recherche irait finalement à prouver qu'il ne faut pas se contenter de l'opinion assez répandue selon laquelle l'homme médiéval, dans sa quête de sécurité, aurait placé d'un côté la Vierge, les saints, tenus pour "sécurisants » et légitimant un certain nombre de pratiques rassurantes, et de l'autre Dieu lui-même (Le Père, la Trinité, le Christ) parmi les objets ou personnages inquiétants et redoutés, notamment au moment clé du Jugement. L'examen même rapide du dossier iconographique que nous avons présenté ne confirme pas, mais infirmerait plutôt, l'idée d'un Dieu terrible. Certaines images de la Trinité, en particulier celles qui comportent le motif du Christ de pitié, ont certainement compté parmi les images plutôt rassurantes de Dieu : c'est du 
moins ce que suggère leur présence en contexte funéraire. Confier le soin d'intercéder aux seuls saints ne procurait au fond aucune certitude: car le résultat de leur intercession demeurait incertain tant que le Juge suprême ne s'était pas prononcé. C'est de la bouche de Dieu lui-même, ou de sa silencieuse et apaisante figure trinitaire, qui enveloppe l'Homme de douleurs, principal gage de miséricorde, que l'on a voulu alors recevoir l'assurance ultime d'être sauvé.

\section{BIBLIOGRAPHIE}

A. AugENENDT, « Theologie und Liturgie des mittelaltÉrichen Toten-Memoria », Memoria, 1984, pp. 79-199.

D. AleXANDRE-Bidon, La Mort au Moyen Âge (XIII ${ }^{e}-\mathrm{XVI}^{e}$ siècle), Paris, 1998.

D. AleXANDRE-Bidon \& C. TREFFoRT (éd.), À Réveiller les morts. La mort au quotidien dans l'Occident médiéval, Lyon, P.U.L., 1993.

Ph. ARIÈs, Essais sur l'histoire de la mort en Occident, du Moyen Âge jusqu'à nos jours, Paris, 1975 ; L'Homme devant la mort, Paris, Le Seuil, 1977 (sp. pp. 213-231 pour les épitaphes); Images de l'homme devant la mort, Paris, Le Seuil, 1983.

J. AVRIL, « La paroisse médiévale et la prière pour les morts ", L'Église et la mémoire des morts dans la France médiévale [table ronde du CNRS, 1982], Paris, 1986, pp. 53-68.

G. BARTZ \& E. KöNIG, « Die Illustration des Totenoffiziums in Stundebüchern », H. Becker, B. Einig, P.-O. Ullrich (éd.), Angesicht des Todes. Ein interdisziplinäres Kompendium, Sankt Ottilien, t. 1, 1987, pp. 487-528.

K. BAUCH, Das mittelalterliche Grabbild. Figürliche Grabmäler des 11. bis 15. Jahrhundert in Europa, Berlin/New York, 1976 (sp. chap. XV : « Epitaphien », pp. 198-214).

Fr. BœSPFLUG, « La Double Intercession en procès. De quelques effets iconographiques de la théologie de Luther ", F. Muller (éd.), Art, religion et société dans l'espace germanique au XVI siècle (colloque de Strasbourg, mai 1993), Strasbourg, Presses universitaires de Strasbourg, 1997, pp. 31-61.

- La Trinité dans l'art d'Occident (1400-1460). Sept chefs-d'œuvre de la peinture, Strasbourg, 2000.

Fr. BæSPFLUG \& Y. ZALUSKA, « Le dogme trinitaire et l'essor de son iconographie en Occident de l'époque carolingienne au IV ${ }^{\mathrm{e}}$ Concile du Latran (1215) », Cahiers de Civilisation médiévale XXXVII, 1994, pp. 181-240

K. BREHM (éd.) et alii, Grabmalkunst aus vier Jahrhunderten : Epitaphien und Grabdenkmäler in der Nikolaikirche zu Berlin: Katalog der Sepulkralplastik, Berlin, 1994 (toutes les épitaphes cataloguées sont postérieures à 1550).

D. BRIESEMEISTER, Bilder des Todes, Untersneidheim, 1970.

F. BURGER, Geschichte des florentinischen Grabmals, Strasbourg, 1904.

Manuel SANCHEZ CAMARGO, La muerta y la pintura espanola, Madrid, 1954. 
J. CHIFFOLEAU, «Sur l'usage obsessionnel de la messe pour les morts à la fin du Moyen Âge », Faire croire. Modalités de la diffusion et de la réception des messages religieux du XII au XVe siècle, Rome, 1981, pp. 236-256.

P. CLEMEN, Belgische Kunstdenkmâler, Munich, 2 t., 1923.

Death in the Middle Ages, Leuven 1983.

L. DIMIER, Les Danses macabres et l'idée de la mort dans l'art chrétien, Paris, 1902.

H. F. O. Evans, « The Holy Trinity on Brasses », Transactions of the Monumental Brasse Society, 13, 1982/3, pp. 208-223.

R. FAVREAU, «Fonctions et inscriptions au Moyen Âge », Cahiers de civilisation médiévale, 1989, 32, pp. 203-232.

R. Favreau, J. Michaud et B. Mora, Corpus des inscriptions de la France médiévale,19, Jura, Nièvre, Saône-et-Loire, Paris, 1997.

P.-A. FÉVRIER, « Quelques aspects de la prière pour les morts », La prière au Moyen Âge (littérature et civilisation), Aix-en-Provence/Paris (« Sénéfiance », 10), pp. 255-282.

- La figuration des morts dans l'Occident médiéval jusqu'à la fin du premier quart du XIV siècle, Fontevraud, 1989.

W. H. FORSYTH, The Entombment of Christ. French Sculptures of the Fifteenth and Sixteenth Centuries, Cambridge (Mass.), 1970.

E. Galletier, G. Bernt, D. Coppini, W. HörAndner, « Epitaphium », Lexikon des Mittelalters, t. 3, 1986.

Grabmalkunst aus vier Jahrhunderten, cat. d'expo Berlin, 1994 (voir Brehm).

W. GRAF, Christliche Grabmalsymbole. Eine Untersuchung auf dem Basel-Städtischen Friedhof am Hörnli, Bâle, 1983.

J. HARASIMOWicz, Mors janua coeli, Wroclaw, 1992.

E. HeRTLEIN, Massacios Trinität. Kunst, Geschichte und Politik der Frührenaissance in Florenz, Florence, 1979.

B. G. LANE, « The « Symbolic Crucifixion », the Hours of Catherine of Cleves », Oud Holland, 87, 1973, pp. 4-26.

M. LAUWERS, La mémoire des ancêtres, le souci des morts. Morts, rites et sociétés au Moyen Âge, Paris, Beauchesne, 1996.

J.-L. Lemaître (éd.), L'Église et la mémoire des morts dans la France médiévale [Table-ronde du CNRS], Paris, 1986.

M. LuTZE, Das plastische Bildepitaph in Deutschland, diss., Leipzig, 1931.

M. MEISs, « La mort et l'office des morts à l'époque du Maître de Boucicaut et des Limbourg », Revue de l'art, 1, 1968, pp. 127-148.

- La peinture à Florence et à Sienne après la Peste noire. Les arts, la religion, la société au milieu du XIV siècle, tr. fr., préface de G. Didi-Huberman, Paris, Hazan, 1994.

Memoria. Der geschichtliche Zeugniswert des liturgischen Gedenkens im Mittelalter, K. Schmid et J. Wollasch (éd.), Munich, 1984.

La Mort et l'au-delà en France méridionale (XII ${ }^{e}-X V^{e}$ siècles), "Cahiers de Fanjeaux 》, n 33, Fanjeaux, Toulouse, Privat, 1998. 
I. MOSNERON-Dupin, « La Trinité debout en ivoire de Houston et les Trinités bourguignonnes de Jean de Marville à Jean de la Huerta », Wiener Jahrbuch für Kunstgeschichte, 43, 1990, pp. 35-65.

G. NeumAnN, Die Ikonographie des Gnadenstuhls, thèse (philosophie), Univ. Libre de Berlin, déc. 1952.

J. NTEDIKA, L'Évocation de l'au-delà dans la prière pour les morts. Études de patristique et de liturgie latines (IVe-VIII ${ }^{e}$ siècle), Louvain/Paris, 1971.

C. C. OLDS, Ars moriendi : a Study of the Form and Contents of Fifteenth-centiry Illustrations of the Art of Dying, thèse dact., 1966

E. PANOFSKY, Tomb Sculpture : four Lectures on its changings aspects from Ancient Egypt to Bernini, New York [conférences données à l'automne 1956], s. d. ; tr. fr : La Sculpture funéraire, de l'Égypte ancienne au Bernin, avant-propos de Martin Warnke, Paris, Flammarion, 1995.

- «Imago pietatis. Contribution à l'histoire des types du « Christ de Pitié »/« Homme de Douleurs » et de la « Maria Mediatrix », Peinture et dévotion en Europe du Nord à la fin du Moyen Âge, Paris, 1997, pp. 13-28.

S. J. PEARMAn, The iconographic Development of the Cruciform Throne of Grace from the Twelfth to the Sixteenth Century, thèse, Cas Western Reserve, 1974.

H.-R. PHILIPPEAU, « Introduction à l'étude des rites funéraires et de la liturgie des morts », La Maison-Dieu, $\mathrm{n}^{\circ}$ 1, 1945, pp. 37-63.

Prier au Moyen Âge. Pratiques et expériences ( $v^{e}-X V^{e}$ siècle), Turnhout, Brepols, 1991.

Fr. RAPP, L'Église et la vie religieuse en Occident à la fin du Moyen Âge, Paris, P.U.F., 1971 (sp. p. 152 s. : « La peur de l'au-delà : l'art de bien mourir »).

É. RAUNIÉ, Épitaphier du vieux Paris : recueil général des inscriptions funéraires des églises, couvents, collèges, hospices, cimetières et charniers, depuis le moyen âge jusqu'à la fin du XVIII ${ }^{e}$ siècle, Paris, 1890 et suiv.

É. REBILLARD, «In hora mortis ». Évolution de la pastorale chrétienne de la mort aux IVe et $V^{e}$ siècles dans l'Occident latin, Rome, 1994.

G. RING, « Beiträge zur Plastik von Tournai im 15. Jhdt. », in P. Clemen, t. 1, p. 269-291 (avec un échantillon de 31 épitaphes qui n'a pas valeur d'inventaire exhaustif, et ne fournit malheureusement pas les légendes).

H. M. RoE, « Illustrations of the Holy Trinity in Ireland, $13^{\text {th }}$ to $17^{\text {th }}$ Centuries ", Journal of the Royal Society of the Antiquiries of Ireland, 109, 1979, pp. 101-150.

P. Rolland, Les Primitifs tournaisiens. Peintres et sculpteurs, Bruxelles/Paris, 1932.

D. SICARD, La liturgie de la mort dans l'Église latine, des origines à la réforme carolingienne, Münster, 1978.

O. voN SIMSON, « Über die Bedeutung von Masaccios Trinitätsfresko in Santa Maria Novella », Jahrbuch der berliner Museen, N.F., t. 8, 1966, pp. 119-158.

J. C. SMITH, German Sculpture of the Later Renaissance, c. 1520-1580, in a Age of Uncertainty, Princeton, 1994 (chap. 5 : «In Memoriam : Epitaphs and Simple Tombs »; chap. 6 : «Commemorative Series and Complex Tombs»).

M. STIRM, « Les images et la Bible », dans G. Bedouelle \& B. Roussel (dir.), Le Temps des Réformes et la Bible, « Bible de tous les temps, 5 », Paris, Beauchesne, 1989, pp. 683-750.

A. TENENTI, La Vie et la mort à travers l'art du XVe siècle, Paris, 1952. 
R. et M. TENNENHAus, Das unsterbliche Bildnis. Europäische gravierte Metallgrabplatten vom 12. bis zum 17. Jahrhundert, Vienne, 1977.

G. TöRÖK, « Beiträge zur Verbreitung einer niederländischen Dreifaltigkeitsdarstellung im 15. Jahrhundert. Eine Elfenbeintafel aus dem Besitze Philipps des Guten von Burgund », Jahrbuch der Kunsthistorischen Sammlungen in Wien, 81, 1985, pp. 7-31.

C. TREFFORT, Genèse du cimetière chrétien. Étude sur l'accompagnement du mourant, les funérailles, la commémoration des défunts et les lieux d'inhumation à l'époque carolingienne (entre Loire et Rhin, milieu VIII ${ }^{e}$-déb. XI ${ }^{e}$ siècle), Thèse, Lyon-II, 1994.

W. VöGE, « Nicolaus von Leyden's Strassburger Epitaph und die holländische Steinplastik, », Oberrheinische Kunst, IV, 1929-1930, p. 35 s.

M. Vovelle, La Mort et l'Occident, de 1300 à nos jours, Paris, Gallimard, 1983.

A. WECKWERTH, « Der Ursprung des Bildepitaphs », Zeitschrift für Kunstgeschichte, 20/1, 1957, pp. $147-185$.

J. WIRTH, La jeune fille et la mort. Recherches sur les thèmes macabres dans l'art germanique de la Renaissance, Genève, 1979.

K. WollasCH, «Les Moines et la mémoire des morts », Religion et culture autour de l'an mil. Royaume capétien et Lotharingie, Paris, Picard, 1990, pp. 47-54.

\section{NOTES}

1. M. Meiss, «La mort et l'office des morts à l'époque du Maître de Boucicaut et des Limbourg », Revue de l'art, 1968/1-2, pp. 17-25.

2. J. Chiffoleau, dans Faire croire, pp. 235-256, sp. 250. Les titres complets des ouvrages cités en abrégé dans les notes se trouvent dans la bibliographie à la fin de cet article.

3. É. Mâle, Fin, sp. pp. 406-410.

4. Paris, BNF, fr. 9471, f. $159^{\mathrm{r}}$ (avec, sur la banderole sortant de la bouche du défunt ou de l'agonisant, la prière In manus tuas; Dieu réponds en français, de manière plutôt rassurante : "Pour tes péchés pénitence feras ; au jour du jugement, avecque moi seras ») et $135^{\mathrm{r}}$ (heures de la Croix).

5. Paris, BNF, lat. 18014, f. $198^{\mathrm{r}}$; V. Leroquais, Livres d'heures, t. 1, p. 175s. ; Simson, fig. 11 p. 134 et p. 138 ; Török, p. 22. Voici le texte de la prière : «Sainte benoite Trinité, trois Personnes en un Dieu, et un Dieu en trois Personnes, le Père, le Fils et le Saint-Esprit, qui êtes le fondement de la foy de sainte eglise, veuillez moy donner grâce de toujours tenir de vous fermement cette foy et cette créance et d'avoir et de mettre en vous toute mon espérance et d'être conjoint à vous par vraie charité et parfaite alliance jusqu'au point de la mort en telle manière que je vous puisse voir face à face avec vos saints anges en votre perdurable gloire, Amen. " Je n'ai pas pu consulter le volume de commentaire du fac similé, sous la direction de Fr. Avril.

6. Trône de grâce, Compassion du Père, Pressoir mystique et Double Intercession, mais aussi Trinité du Psautier, Trinité triandrique et Couronnement de la Vierge par la Trinité.

7. Aussi ancien, voire antérieur de peu, est le relief d'Aguilar de Campoo (Palencia) conservé au Musée archéologique national de Madrid, sur le sarcophage d'un abbé du monastère prémontré de Santa Maria la Real ; voir G. De Pamplona, Iconografia de la santisima Trinidad en el arte medieval espanol, Madrid, 1970, fig. 37 et p. 99. 
8. Lutze, pp. 22-25, y voit une étape vers le Bildepitaph; Weckwerth, p.169, n.144; Hertlein, p. 81-82, en parle comme d'une pierre tombale (Grabstein), et y voit le plus ancien monument funéraire avec Trinité.

9. Neumann, p. 37.

10. Pearmann, $\mathrm{n}^{\circ} 354$ et p. 113.

11. Repr. in «Les anciennes industries tournaisiennes d'art. La sculpture », Bulletin des métiers d'art, t. XII, 1913, pp. 42-54 (50).

12. Munich, Bayerisches Nationalmusem (v. 1435) ; Neumann, p. 51 ; Hertlein, fig. 50 et p. 97 ; K. Bauch, Das mittelalterliche Grabbild, Berlin/New York, 1976, p. 209 s.; Fr. Bœspflug, «La (com)passion de la Trinité dans l'art flamand $\mathrm{du} \mathrm{xv}^{\mathrm{e}}$ siècle ", in P. Gésché (éd.), Dieu à l'épreuve de notre cri, Paris, Cerf, 1999, pp. 45-67 ; Das Goldene Roessl. Ein Meisterwerk der Pariser Hofkunst um 1400, cat. d'expo, Munich, Bayerisches National Museum, 1995, cat. n 7, p. 226.

13. Lutze, p. $36 \mathrm{~s}$.

14. Roe, fig. 42 , p. 126 et $n^{\circ} 21$ p. 142.

15. Roe, fig. 29a p. 113 et notice 7 p. 133.

16. L'une des plus anciennes épitaphes au sens défini plus haut, qui n'a pas de motif trinitaire, est une dalle gravée de l'église du château d'Iburg, près d'Osnabrück (vers 1345): Alemung von Varendorp $(\dagger 1355)$ et sa femme Amalberga († 1345) sont agenouillés devant la croix (une croix nue), avec leurs écus ; Bauch, fig. 311, p. 199.

17. Bauch, p. 198.

18. Bauch, p. 198.

19. Ariès, L'Homme devant la mort, p. 230 s. n'est pas très clair à ce sujet, et parle, non d'épitaphe mais de «tombeau-épitaphe": «une petite plaque d'environ $20 / 30 \times 40 / 50 \mathrm{~cm}$, entièrement occupée par une inscription, sans autre figure »; ce que les chercheurs allemands appellent Epitaph semble correspondre à ce qu'Ariès nomme «tableau ", p. 271 s., distinct de la tombe, ou "plaque murale», p. 276; le livre de Bauch paraît mieux distinguer les différents objets en question.

20. Panofsky, La Sculpture funéraire, tr . fr., p. 70.

21. R. Favreau, «Fonctions des inscriptions au Moyen Âge ", Cahiers de civilisation médiévale, 1989, 32, pp. 203-232.

22. Panofsky, La Sculpture funéraire, fig. 197 (Jean Fiévez, † 1425).

23. Sur la représentation des donateurs dans l'art médiéval, je n'ai pas eu accès à D. Kocks, Die Stifterdarstellung in der italienischen Malerei des 13.-15. Jahrhunderts, diss., Cologne, 1971.

24. Panofsky, La Sculpture funéraire, fig. 200 (Tournai, cathédrale).

25. Bauch, fig. 309.

26. Bauch, fig. 319, p. 205 (Siegfried zum Pardies, $\uparrow 1386$ ).

27. Rolland, fig. 22.

28. Ainsi dans la stèle funéraire du Chanoine de Wastine († 1433); Rolland, fig. 19.

29. Rolland, fig. 29-31; Bauch, fig. 323 (Étienne Yvert, chanoine de N.-D. de Paris, † 1462).

30. Rolland, fig. 5, 10, 11, 13) ; Clemen, t. 1, pl. 36 (Simon de Leval, s. d.), fig. 290 (G. Parent, s. d.), 292 (Baudouin de Hénin, † 1420), 293 (Maire de Qinghien, † 1427), 298 (Jehan du Bos, † 1438), 300 (Jacquemart Hainecart, $\uparrow 1434)$.

31. Rolland, fig. 15.

32. Lane, p. 16 (biblio.) en parle comme d'une épitaphe, p. 17 ; Hertlein, n. 217 p. 76 ; Bauch, fig. 328 et p. 212 s. ; Koepplin, in Martin Luther und die Reformation in Deutschland, $n^{\circ} 446$.

33. Sur ce type iconographique, qui s'inspire d'Arnaud de Chartres, un disciple de Bernard de Clairvaux, et qui fait son apparition précisément à cette époque, voir D. Koepplin et Fr. Bœspflug, « La Double Intercession en procès ».

34. Te rogo virgo pia nunc me defende maria/ hec quia sucsisti fili veniam precor isti / vulnera cerne pater fac comme rogetat mea mater / queque petita dabo fili tibi nulla negabo. 
35. Docliximo Figliuolo pellacte chio tidie abbi mia [=misÉricordia] di chostoro / Padre moi sieno salvi chostoro pequali tu volesti chio patissi passione.

36. Lane, p. 17 n. 69, fournit la liste des principales attributions; Hertlein, p. 40. Superbe reproduction en couleur et en pleine page dans La Légende dorée, Paris, Diane de Selliers Éditeur, 2000 , t. 2, p. 241.

37. Bœspflug, La Trinité, pp.103-125, sp.115-117. Avant cette fresque, il se pourrait que la multiplication des panneaux peints et retables toscans avec le Trône de grâce comme motif central ait quelque rapport à la mort et à la Peste noire qui commence de se répandre en 1349 .

38. Simson, p. 138.

39. Clemen, t. 1, p. 270 ; Stirm, p. 686.

40. Ces monuments ont été étudiés surtout dans une perspective purement formelle, pour les liens de ces panneaux en relief avec les panneaux peints contemporains, et dans le cadre de la discussion sur l'identité de Van der Weyden: ainsi chez C. Clemen, Belgische Kunstdenkmäler, Munich, 1923 ; de même chez Rolland, p. 64-65 et fig. 9, 46 et 47 (le réalisme des portraits de donateurs conduit l'A. à y voir le reflet direct de l'œuvre peint du Maître de Flémalle, alias Roger de la Pasture); Simson, p. 129 s. et 138 ; J.-G. Ghislain, Les grands siècles de Tournai, Tournai/ Louvain-la-Neuve, 1993, pp. 115-208.

41. Ph. Ariès, Images, p. 79.

42. Clemen, t. 1, fig. 299 ; Hertlein, fig. 49.

43. Hertlein, fig. 48 ; G. Ring, dans P. Clemen, t. 1 , n² 29 p. 276 et p. 278 ; Fr. Van der Meer et H. Sibbelee, Images du Christ dans la sculpture au Nord des Alpes et des Pyrénées, $n^{\circ} 154$, Anvers/Paris, 1980.

44. Clemen, t. 1, fig. 294.

45. Clemen, t. 1, pl. 294, et $\mathrm{n}^{\circ} 20$ p. 276 ; Pearman, $\mathrm{n}^{\circ} 357$; Simson, p. 136 s.

46. Clemen, t. 1, fig. 297 p. 284 et $n^{\circ} 9$ p. 273.

47. Clemen, t. 1, pl. 37.

48. Fr. Gorissen, Das Stundebuch der Katharina von Kleve. Analyse und Kommentar, Berlin, 1973, fig. 475 p. 993.

49. Clemen, t. 1, fig. 291.

50. Ariès, Images, p. 78.

51. A. Tenenti; C. C. Olds, Ars moriendi: a Study of the Form and Contents of Fifteenth-century Illustration of the Art of Dying, thèse, Pa, 1966; Ariès, Images, p. 156-157 ; M. Lauwers, "Ars moriendi », in A. Vauchez (dir.), Dictionnaire encyclopédique du Moyen Âge, Paris, 1997.

52. En particulier dans la gravure illustrant la «Tentation de vaine gloire »: Tenenti, p. 55, 99, 101, 109, 111, 113.

53. Pour ce groupe d'images, voir dans la bibliographie Koepplin, et Bœspflug, «La Double Intercession ».

54. Cat. d'expo. Manuscrits à peintures du XIII ${ }^{e}$ au XVI siècle, Paris, Bibliothèque nationale, 1955, $\mathrm{n}^{\circ} 115$, p. 57 ; déjà Leroquais, Psautiers, t. 1, p. 67, avait signalé cette miniature. Une composition de même type, près d'un demi-siècle plus tard, en 1388, se trouve dans une fresque de Zseliz, près d'Esztergom (Hongrie) : J. Végh, « The Particular Judgement of a Courtier : a Hungarian Fresco of a Rare Iconographical Type ", Arte Cristiana, t. 74, 1986, p. 303-314 ; J. Baschet, "Jugement de l'âme, jugement particulier : contradiction, complémentarité, chevauchement ", Revue Mabillon, 6 (= t. 67), 1995, pp. 159-203, fig. 4 et p. 179.

55. Florence, BN, Codex B.R. 38; W. Cohn, «Eine Unbekannte Oberrheinische Miniatur des « weichen Styls » ", Festschrift Friedrich Winkler, Berlin, 1959, pp. 95-99 ; cat. d'expo. Martin Luther und die Reformation in Deutschland, 1983, $n^{\circ}$ 447, p. 338 (D. Koepplin).

56. Dans un recueil cartusien de la première moitié $d u \mathrm{Xv}^{\mathrm{e}}$ siècle, un dessin à la plume représente la même dispute au chevet d'un mourant: Londres, BL, Add. Ms. 37049, f. 19 ; J. Hogg, «An 
Illustrated Yorkshire Carthusian Religious Miscellany British Library London Additional Ms 37049 ", Analecta Cartusiana, Salzburg, vol. 3, p. 20.

57. Cracovie, Archiwum i Biblioteka Kapituly Metropolitanij na Wawelu, Missel $\mathrm{n}^{\circ} 2$, KP,

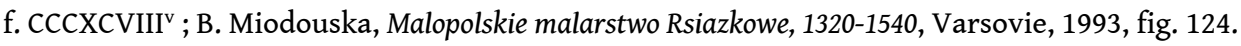

58. Ariès, Images, p. 258, reproduit un bois gravé bourguignon du XVII ${ }^{\mathrm{e}}$ siècle qui est la copie d'un tableau (« La Bonne mort ») de l'église Saint-Prix-et-Saint-Lot de Saint-Bris (Yonne) ; voir Trésors d'art des Églises de l'Yonne, t. 2, Paris, 1971, n² 25.

59. Leipzig, Museum der bildenden Künste, Inv. Nr. 1924. 40 ; Neumann, p. 68 s. ; D. Koepplin et T. Falk, Lucas Cranach, cat. d'expo. de Bâle, 1974, Bâle/Stuttgart, 1974, t. 2, p. 466 s. ; Stirm, p. 720 , n. 107 (repr. p. 722) ; cat. d'expo. Lucas Cranach, 1994, A 108, fig. 163.

60. Dans le même sens, voir aussi la gravure de Lucas Cranach l'A. sur l'Échelle de saint Bonaventure, Paris, BNF ; Est., Ca 9 rés.; M. Hébert, Inventaire des gravures des écoles du Nord, 1440-1550, Paris, t. 1, 1982, nº 886 (antérieure : 1506), une autre gravure de 1509 (ibid., n 901, 17), puis trois tableaux, l'un de 1515, conservé à Leipzig, un autre de 1516-18, à Cobourg, et le troisième, de 1530 (?), à Brême.

61. Neumann, p. 75 s. ; Pearmann, p. 95 ; L. H. Heydenreich « Ein In-Memoriam Bild des Jacopo del Sellaio ", Die Kunst und das schöne Heim, vol. 50, avril 1952 ; M. Gregorio et S. Blasso, Firenze nella pittura e nel designo dal Trecento al Settecento, Milan, 1994, p. 64 et fig. 63 ; N. Pons, "Una provenienza per Jacopo del Sellaio ", Antichita viva, 33, 1994/6, pp. 16-19.

62. Fr. Bœspflug, «La Trinité dans l'art avant 800 », Le Monde de la Bible, n 110, avril 1998, pp. 79-87.

63. C'est du moins une hypothèse que permettent d'envisager les dalles funéraires d'Afrique comportant un monogramme enveloppé ou couronné d'un triangle, sans que l'on soit parvenu à une certitude à ce sujet ; voir J.-A. Martigny, Dictionnaire de l'Antiquité chrétienne, 2è édition, 1977, p. 766, cité par J. J. M. Timmers, « Dreieck », LCI, t. 1, col. 325. La présence de Dieu le Père flanqué de son Logos sur le Sarcophage des Époux du Musée d'Arles évoque l'action créatrice de Dieu mais n'est jamais qu'un motif parmi beaucoup d'autres, et ne signifie sans doute pas que les défunts aient entendu se placer sous la protection spéciale de la Trinité.

64. En contexte protestant, voir les deux épitaphes de 1598 et 1602 in Scharfe, n. 1 p. 102 ; pour la Silésie des XVI ${ }^{\mathrm{e}}$ et XVII ${ }^{\mathrm{e}}$ siècles, voir Harasimowicz, 1992, fig. 6, 20, 43 (Crucifixion trinitaire). Mais la Réforme tentera de mettre un terme à tout le système de protection, et en particulier à l'usage « délirant » des vieux moyens d'intercession (Chiffoleau, p. 255).

65. Voir Ariès, Images de l'homme devant la mort.

66. W. Graf, Christliche Grabmalsymbole. Eine Untersuchung auf dem Basel-Städtischen Friedhof am Hörnli, Bâle, 1983, pp. 54-58 (quelques exemples du xx siècle).

67. Psautier de sainte Élisabeth, École thuringo-saxonne, Cividale, av. 1217 ; Fr. Bœspflug-Y. Zaluska, pl. III d ; parmi tant d'autres exemples ultérieurs, mentionnons seulement la superbe enluminure des Statuts de l'Ordre du Saint Esprit (Paris, BNF, fr. 4274, fol. $2^{\mathrm{v}}$; 1354-55 ; Hertlein, p. 163), celle des Heures de Jeanne de Navarre (Paris, BNF, n.a.l. 3145 , fol. $3^{v}$; v. 1420) et enfin celle des Heures de Louis de Savoie (Paris, BNF, lat. 9473, fol. 141 ${ }^{\mathrm{r}}$ ).

68. Jacques Le Goff, La Civilisation de l'Occident médiéval, Paris, 1982, p. 128 : « le thème trinitaire semble surtout avoir exercé son attrait sur les milieux théologiques savants et n'avoir eu qu'un retentissement limité dans les masses. »

69. Le nombre de messes demandées, par exemple trois ou trente, dans les testaments nobles et royaux, déjà au XIII ${ }^{\mathrm{e}}$ siècles, pourrait avoir quelque rapport à la Trinité ; voir le chapitre que Guillaume Durand consacre à l'office des morts dans son Rational des divins offices (1. 6, chap. 35, cité par Arasse, p. 238 s.).

70. Cité par Pearmann, p. 93.

71. Le témoignage le mieux connu, et le plus visible, de cette pratique est offert par les « colonnes de peste » (Pestsaülen) qui ont été dressées dans bon nombre de villes de l'Europe du centre et de 
l'est aux XVII ${ }^{e}$ et XVIII ${ }^{e}$ siècles pour remercier Dieu de l'éloignement du fléau : la plupart d'entre elles étaient surmontées d'un groupe sculpté de la Trinité et constituaient de la sorte un acte public par lequel les cités se plaçaient sous la protection de la Trinité et lui exprimaient leur gratitude pour avoir été miraculeusement préservées. Sur les colonnes de peste surmontées d'une Trinité, voir Pearmann, p. 100.

72. Sur les gonfalons anti-pesteux, voir D. Arasse, «Entre dévotion et culture: fonctions de l'image religieuse au xv siècle », dans Faire croire, pp. 131-146 (141 s.) : mais les exemples étudiés par cet auteur n'ont pas le thème trinitaire. C'est généralement Marie (assistée de saints) qui, par son manteau et/ou son intercession, protège des flèches de peste décochées par le Christ ou Dieu le Père.

73. Dans la fresque de San Gimignano peinte par Gozzoli en 1464, saint Sébastien protège de son manteau (contre lequel elles se cassent) tout un peuple des flèches de peste décochées par Dieu le Père, dont la double intercession de Marie montrant ses seins et du Christ montrant sa plaie cherche à suspendre le geste meurtrier ; voir M. Opitz, Benozzo Gozzoli, 1420-1497, Bonn, 1998, p. 90. 74. Panofsky, Imago pietatis, p. 284 et 289.

75. Eja ergo, advocata nostra, illos tuos misÉricordes oculos, ad nos converte.

76. D'après H. Leclercq, « Marie (Je vous salue) », Dictionnaire d'archéologie chrétienne et de liturgie, t. 10, 1931, col. 2043-2062 (2058-61), la dernière partie de la Salutation angélique ne semble pas antérieure $\mathrm{au} \mathrm{XV}^{\mathrm{e}}$ siècle et ne se généralise pas avant la fin $\mathrm{du} \mathrm{XVI}^{\mathrm{e}}$ siècle ; J. Toussaert, Le Sentiment religieux en Flandre à la fin du Moyen Âge, Paris, 1963, p. 347, parvient à la même conclusion.

77. Sans doute cette prière fut-elle comprise au Moyen Âge comme une demande concernant spécifiquement la grâce de la bonne mort, plutôt que l'assistance de Marie au-delà de la mort. Mais il est probable que l'on ait espéré les deux d'une telle prière.

\section{AUTEUR}

\section{FRANÇOIS BEESPFLUG}

Faculté de théologie catholique, Université Marc-Bloch, Strasbourg 